\title{
Retinol-binding protein 4 is associated with components of the metabolic syndrome, but not with insulin resistance, in men with type 2 diabetes or coronary artery disease
}

\author{
M. von Eynatten • P. M. Lepper • D. Liu • K. Lang • \\ M. Baumann • P. P. Nawroth • A. Bierhaus • \\ K. A. Dugi • U. Heemann • B. Allolio • P. M. Humpert
}

Received: 20 April 2007 / Accepted: 25 May 2007 / Published online: 18 July 2007

(C) Springer-Verlag 2007

\begin{abstract}
Aims/hypothesis Retinol-binding protein 4 (RBP4) has recently been reported to be associated with insulin resistance and the metabolic syndrome. This study tested the hypothesis that RBP4 is a marker of insulin resistance and the metabolic syndrome in patients with type 2 diabetes or coronary artery disease (CAD) or in non-diabetic control subjects without CAD.

Methods Serum RBP4 was measured in 365 men (126 with type 2 diabetes, 143 with CAD and 96 control subjects) and correlated with the homeostasis model assessment of insulin resistance index (HOMA-IR), components of the metabolic syndrome and lipoprotein metabolism. RBP4 was detected by ELISA and validated by quantitative Western blotting.

Results RBP4 concentrations detected by ELISA were shown to be strongly associated with the results gained in quantitative Western blots. There were no associations of
\end{abstract}

M. von Eynatten $(\bowtie) \cdot$ D. Liu $\cdot$ M. Baumann $\cdot$ U. Heemann

Department of Nephrology, Technical University Munich,

Ismaningerstr. 22,

81675 Munich, Germany

e-mail: maximilian.eynatten@1rz.tum.de

M. von Eynatten $\cdot$ K. Lang $\cdot$ B. Allolio

Department of Medicine I, Wuerzburg University,

Wuerzburg, Germany

P. M. Lepper

Department of Intensive Care Medicine,

University of Bern (Inselspital),

Bern, Switzerland

P. P. Nawroth · A. Bierhaus · K. A. Dugi • P. M. Humpert Department of Medicine I and Clinical Chemistry,

Heidelberg University,

Heidelberg, Germany
RBP4 with HOMA-IR or $\mathrm{HbA}_{1 \mathrm{c}}$ in any of the groups studied. In patients with type 2 diabetes there were significant positive correlations of RBP4 with total cholesterol, LDL-cholesterol, VLDL-cholesterol, plasma triacylglycerol and hepatic lipase activity. In patients with CAD, there were significant associations of RBP4 with VLDLcholesterol, plasma triacylglycerol and hepatic lipase activity, while non-diabetic control subjects without CAD showed positive correlations of RBP4 with VLDLcholesterol and plasma triacylglycerol.

Conclusions/interpretation RBP4 does not seem to be a valuable marker for identification of the metabolic syndrome or insulin resistance in male patients with type 2 diabetes or CAD. Independent associations of RBP4 with pro-atherogenic lipoproteins and enzymes of lipoprotein metabolism indicate a possible role of RBP4 in lipid metabolism.

Keywords Adipokines - Coronary artery disease .

Hepatic lipase - Insulin resistance · Lipoprotein ·

Lipoprotein lipase $\cdot$ Lipoprotein metabolism .

Metabolic syndrome $\cdot$ RBP $4 \cdot$ Retinol-binding protein 4 .

Type 2 diabetes

\begin{tabular}{ll}
\multicolumn{2}{l}{ Abbreviations } \\
apo & $\begin{array}{l}\text { apolipoprotein } \\
\text { CAD }\end{array}$ \\
coronary artery disease \\
hOMA-IR & $\begin{array}{l}\text { homeostasis model assessment of insulin } \\
\text { resistance } \\
\text { retinol-binding protein } 4\end{array}$
\end{tabular}

\section{Introduction}

The adipokine retinol-binding protein 4 (RBP4) was recently reported to be associated with insulin resistance 
and components of the metabolic syndrome in subjects with type 2 diabetes mellitus, a family history of diabetes, obesity or impaired glucose tolerance [1]. The underlying mechanism for this association seems to be a downregulation of the adipocyte GLUT4 (solute carrier family 2 [facilitated glucose transporter], member 4) in insulinresistant states, leading to an increased secretion of RBP4 [2]. Since downregulation of GLUT4 in adipocytes and RBP4 itself seem to contribute to the development of insulin resistance [2-4], serum RBP4 might be a useful marker for identifying patients affected by the metabolic syndrome, who are known to be at risk of cardiovascular events and premature death $[5,6]$. To test this hypothesis, we quantified RBP4 in 126 men with type 2 diabetes, 143 men with coronary artery disease (CAD) and 96 nondiabetic control subjects without $\mathrm{CAD}$, correlating levels of the adipokine with serum lipids and components of the metabolic syndrome.

\section{Methods}

Patients A total of 365 subjects were included in this study. Three different cohorts of male patients were studied: (1) 126 individuals with type 2 diabetes; (2) 143 patients with CAD; and (3) 96 control subjects without history of CAD or type 2 diabetes. All patients included in this study were recruited out of clinical routine procedures at the University Hospital Heidelberg, Department of Medicine, according to the guidelines of the local ethics committee and after giving written informed consent. CAD was diagnosed angiographically and defined as a stenosis of greater than $50 \%$ of the luminal diameter in at least one of the coronary arteries or their major branches. Type 2 diabetes was defined according to the criteria of the American Diabetes Association [7]. A fasting plasma glucose level of $\geq 7.0 \mathrm{mmol} / 1$ was an exclusion criterion for the CAD and control groups. In all three groups, contraindications for heparin treatment, application of subcutaneous or intravenous heparin in the previous $72 \mathrm{~h}$, severe kidney or liver disease and a fasting triacylglycerol level of $>11.4 \mathrm{mmol} / 1(1,000 \mathrm{mg} / \mathrm{dl})$ (suggestive of secondary lipid disorders) were exclusion criteria.

Quantitative Western blotting of serum RBP4 Modified immunoblotting was performed in 40 patients included in this study (10 patients with type 2 diabetes, 10 non-insulinresistant CAD patients, 10 insulin-resistant $C A D$ patients and 10 control subjects) as previously described in detail [8] using human recombinant full-length RBP4 (3378-LC; R\&D Systems, Wiesbaden, Germany) as standard. Standard solutions of $15,30,60$ or $120 \mu \mathrm{g} / \mathrm{ml} \mathrm{RBP4}$ were prepared in Tris-buffered saline containing 1\% NP-40. Standards and sera were diluted 1:200 in SDS-Page. Blots were incubated overnight with the primary antibody (A0040; DAKOCytomation, DAKO USA, Carpinteria, CA, USA) at a dilution of 1:1,000 and for $1 \mathrm{~h}$ at room temperature with a horseradishperoxidase-conjugated secondary antibody (BD Biosciences, San Jose, CA, USA) at 1:2,500. Bands were detected by enzymatic chemiluminescence (Roche Diagnostics, Mannheim, Germany) and quantified with GeneSnap software (Synoptics/Syngene, Frederick, MD, USA).

Analysis of lipids/lipoproteins and routine blood parameters Total cholesterol, HDL-cholesterol and triacylglycerol levels were determined enzymatically in a Synchron LX-20 (Beckman Coulter, Krefeld, Germany). LDL- and VLDLcholesterol were separated by ultracentrifugation in a centrifuge (LM-8 ultra; Beckman Coulter) in $100 \mu \mathrm{l}$ volumes by use of a VT-51.2 rotor (Beckman Coulter).

Venous blood was drawn after an overnight fasting period for the measurement of RBP4, glucose and insulin. Serum RBP4 samples were analysed in duplicate by sandwich ELISA (Immundiagnostik AG, Bensheim, Germany), with serum of four healthy controls being used for interassay variation. The inter- and intra-assay coefficients of variation were both $<5.0 \%$. Serum insulin immunoreactivity was determined in frozen serum by ELISA (CIS bio international, Gif-Sur-Yvette, France). The degree of insulin resistance was estimated by homeostasis model assessment of insulin resistance index (HOMA-IR) according to the method recommended by Levy et al. [9]. Post-heparin lipoprotein lipase and hepatic lipase activity were detected as previously described in detail [10-12]. Briefly, venous blood was drawn into EDTA tubes before and 10 min after intravenous injection of $60 \mathrm{IU}$ of heparin (Braun Melsungen, Melsungen, Germany) per kg body weight, after which lipoprotein lipase and hepatic lipase activities were determined with a triolein/phosphatidylcholine emulsion. Serum adiponectin was analysed in duplicate by ELISA (B-Bridge International, CA, Mountain View, USA).

Statistical analyses Statistical analyses were performed using SPSS software version 11.0 (SPSS, Chicago, IL, USA). Since serum levels of RBP4 showed a skewed distribution, bivariate Spearman correlation coefficients were used to describe the association between RBP4 and the different variables. Comparison between two cohorts of patients was performed by unpaired $t$ test or the MannWhitney $U$ test. In multivariable analysis models, the independent association of RBP4 serum levels with markers of the metabolic syndrome and lipid metabolism were evaluated. The first model including RBP4 as the dependent variable, included age, BMI, LDL, VLDL, history of hypertension, HOMA-IR and $\mathrm{HbA}_{1 \mathrm{c}}$. The current glucose-lowering medication was included as oral medication, insulin therapy or both (Table 1). The second model 
tested age, BMI, LDL, VLDL, as well as post-heparin activity of lipoprotein lipase and hepatic lipase as independent variables. These covariables were all added to the models, either because they were identified as significantly associated with serum RBP4 in this study or because they had been previously described as determinants of serum RBP4. Variables were tested for colinearity (or multicolinearity) and variables with a low tolerance in colinearity statistics, resulting in increased variance inflation factor values, were excluded. Variables that were not normally distributed, such as RBP4, VLDL-cholesterol, triacylglycerol, $\mathrm{HbA}_{1 \mathrm{c}}$ and HOMA-IR, were log-transformed to better approximate normal distributions. Results are expressed as mean \pm SE or median with interquartile range). A $p$ value of less than 0.05 was considered to be statistically significant.

\section{Results}

Baseline characteristics For patients with type 2 diabetes, $\mathrm{CAD}$ and control subjects, baseline characteristics are shown in Table 1. Type 2 diabetic patients had a higher mean BMI, elevated VLDL-cholesterol and triacylglycerol levels, increased fasting plasma glucose, insulin and HOMA-IR, as well as lower HDL-cholesterol and serum adiponectin compared with control subjects. In our patient cohort, the majority of type 2 diabetes patients were treated with insulin (61.9\%). There were, however, no significant differences in fasting glucose and $\mathrm{HbA}_{1 \mathrm{c}}$ between the insulin-treated patients and those on oral glucose-lowering agents (data not shown). Compared with control subjects, CAD patients were older and had lower mean HDLcholesterol and adiponectin levels, as well as increased HOMA-IR and insulin levels. Serum RBP4 levels were significantly lower in patients with CAD than in control subjects and patients with type 2 diabetes (CAD: 27.8 [22.5-33.9] $\mu \mathrm{g} / \mathrm{ml}$ vs controls: 29.8 [25.1-39.0] $\mu \mathrm{g} / \mathrm{ml}$ and vs type 2 diabetes: 29.5 [23.7-42.9] $\mu \mathrm{g} / \mathrm{ml}$; values: median [interquartile range]; $p<0.05$ for both) (Table 1). However, no difference was found between patients with type 2 diabetes and the control group (diabetes: 29.5 [23.7-42.9] $\mu \mathrm{g} / \mathrm{ml}$ vs controls: 29.8 [25.1-39.0] $\mu \mathrm{g} / \mathrm{ml}$ ) (Table 1).

Comparison of RBP4 measurement by ELISA and quantitative Western blot analyses Quantitative Western blots were previously described as the 'gold standard' for quantification of RBP4 [8]. Hence, we used this method to validate the results obtained by sandwich ELISA in our patients and control subjects. Consistent with the previous data [8], serum RBP4 levels detected by western blot were significantly higher than the concentrations obtained by ELISA in all patient groups (Fig. 1b). To compare the performance of the ELISA with quantitative Western blotting, we tested for linearity in regression analyses. In the 40 patients analysed the overall correlation between RBP4 values measured by ELISA and Western blotting was

Table 1 Clinical characteristics of patients with type 2 diabetes mellitus or CAD and of control subjects

\begin{tabular}{|c|c|c|c|}
\hline Factor & Type 2 diabetes $(n=126)$ & CAD patients $(n=143)$ & Control subjects $(n=96)$ \\
\hline Retinol binding protein $4(\mu \mathrm{g} / \mathrm{ml})^{\mathrm{a}}$ & $29.5(23.7-42.9)$ & $27.8(22.5-33.9)^{* * *}$ & $29.8(25.1-39.0)$ \\
\hline Age (years) & $60.2 \pm 9.6$ & $62.0 \pm 9.9^{*}$ & $58.4 \pm 11.7$ \\
\hline BMI $\left(\mathrm{kg} / \mathrm{m}^{2}\right)$ & $28.9 \pm 4.9^{* *}$ & $27.6 \pm 3.3^{*}$ & $26.6 \pm 3.1$ \\
\hline \multicolumn{4}{|l|}{ Cholesterol (mmol/l) } \\
\hline Total & $5.48 \pm 1.24$ & $5.32 \pm 1.18$ & $5.34 \pm 1.39$ \\
\hline HDL & $0.98 \pm 0.28 * *$ & $1.01 \pm 0.26^{*}$ & $1.12 \pm 0.34$ \\
\hline LDL & $3.60 \pm 0.93$ & $3.75 \pm 1.07$ & $3.57 \pm 0.98$ \\
\hline VLDL $^{\mathrm{a}}$ & $0.62(0.44-1.08)^{* * * *}$ & $0.49(0.31-0.72)$ & $0.49(0.23-0.88)$ \\
\hline Triacylglycerol $(\mathrm{mmol} / \mathrm{l})^{\mathrm{a}}$ & $1.71(1.08-2.53)^{* * * *}$ & $1.39(1.06-1.84)$ & $1.30(0.79-2.03)$ \\
\hline Adiponectin $(\mu \mathrm{g} / \mathrm{ml})^{\mathrm{a}}$ & $4.13(2.35-7.12)^{* * * *}$ & $3.75(2.44-6.44) * * * *$ & $5.23(3.44-9.61)$ \\
\hline Fasting plasma glucose $(\mathrm{mmol} / \mathrm{l})$ & $8.7 \pm 2.9^{*}$ & $5.7 \pm 0.8$ & $5.6 \pm 0.6$ \\
\hline HOMA-IR ${ }^{\mathrm{a}}$ & $6.98(4.43-12.02)^{* * * *}$ & $4.73(3.50-6.07) * * * *$ & $2.11(1.45-3.35)$ \\
\hline Insulin $(\mathrm{pmol} / \mathrm{l})^{\mathrm{a}}$ & $20.5(12.0-28.5)^{* * * *}$ & $20.0(15.8-24.0)^{* * * *}$ & $9.0(6.0-13.0)$ \\
\hline $\mathrm{HbA}_{1 \mathrm{c}}(\%)^{\mathrm{a}}$ & $7.3(6.6-8.3)$ & - & - \\
\hline Oral GLT & $n=48(38.1 \%)$ & - & - \\
\hline Insulin therapy & $n=78(61.9 \%)$ & - & - \\
\hline
\end{tabular}

Data are mean $\pm \mathrm{SE}$ or ${ }^{\mathrm{a}}$ median (interquartile range)

GLT, glucose-lowering therapy

$* p<0.05, * * p<0.001$ vs control subjects (unpaired $t$ test)

$* * * p<0.05, * * * * p<0.001$ vs control subjects (Mann-Whitney $U$ test) 
highly significant $(r=0.75 ; p<0.001)$ (Fig. 1c). Likewise, significant correlations were found in subgroup analyses, which were analysed separately in this study, of patients with type 2 diabetes $(n=10 ; r=0.92 ; p<0.001)$, patients with CAD $(n=20 ; r=0.80 ; p<0.001)$ and control subjects $(n=10 ; r=0.70 ; p=0.024)$ (Fig. $1 \mathrm{~d}-\mathrm{f})$.

Associations of RBP4 with markers of insulin sensitivity and the metabolic syndrome Unexpectedly, patients with type 2 diabetes showed no significant bivariate associations between RBP4 and $\mathrm{HbA}_{1 \mathrm{c}}$, HOMA-IR, BMI or age (Fig. 2a-c). Likewise, patients with $\mathrm{CAD}$ showed no associations of RBP4 with HOMA-IR, BMI (Fig. 3a,b) and age (not shown). The same was true for control subjects and the subgroups of patients when RBP4 levels detected by quantitative western blotting were analysed (not shown). In contrast, serum adiponectin, which has previously been described as a valuable marker of insulin sensitivity (reviewed in [13]), significantly correlated with markers of the metabolic syndrome in patients with type 2 diabetes (BMI: $r=-0.288, p=0.004$; HOMA-IR: $r=-0.210$, $p=0.030$; HDL: $r=0.224, p=0.023$; triacylglycerol: $r=-0.341, p<0.001)$ and in patients with CAD (BMI: $r=$ $-0.252, p=0.002$; HOMA-IR: $r=-0.363, p<0.001$; HDL: $r=0.330, p<0.001$; triacylglycerol: $r=-0.182, p=0.030$ ).

Multivariable regression models were used to estimate the partial association between markers of the metabolic syndrome and the serum RBP4 concentration. In diabetes patients, BMI and VLDL-cholesterol levels were significantly and independently associated with RBP4 levels, whereas HOMA-IR, $\mathrm{HbA}_{1 \mathrm{c}}$ and the current glucoselowering medication showed no associations (Table 2). When data from patients with CAD or control subjects were analysed, only VLDL-cholesterol levels remained independently associated with serum RBP4 (Table 2). Interestingly, when VLDL-cholesterol levels in the analyses were replaced by plasma triacylglycerol, there were no independent associations between triacylglycerol and RBP4 in any of the groups (data not shown). Hence, we further investigated possible influences of RBP4 on lipid metabolism.

Associations of RBP4 with lipid metabolism In patients with type 2 diabetes, bivariate analysis revealed a positive correlation of RBP4 levels with total cholesterol $(r=0.344$; $p<0.001)$, LDL-cholesterol $(r=0.254 ; p=0.008)$, VLDLcholesterol $(r=0.349, p<0.001)$, triacylglycerol levels $(r=$ $0.226, p=0.017)$ and hepatic lipase activity $(r=0.211 ; p=$ 0.039) (Fig. 2d-f). Significant associations between RBP4 and markers of lipid metabolism were also present in patients with CAD (VLDL-cholesterol: $r=247 ; p=0.003$; triacylglycerol levels: $r=0.289 ; p<0.001$; hepatic lipase activity: $r=0.174 ; p=0.033$ ) (Fig. $3 \mathrm{c}, \mathrm{d}$ ) and in control subjects (VLDL-cholesterol: $r=0.490 ; p<0.001$; triacyl-

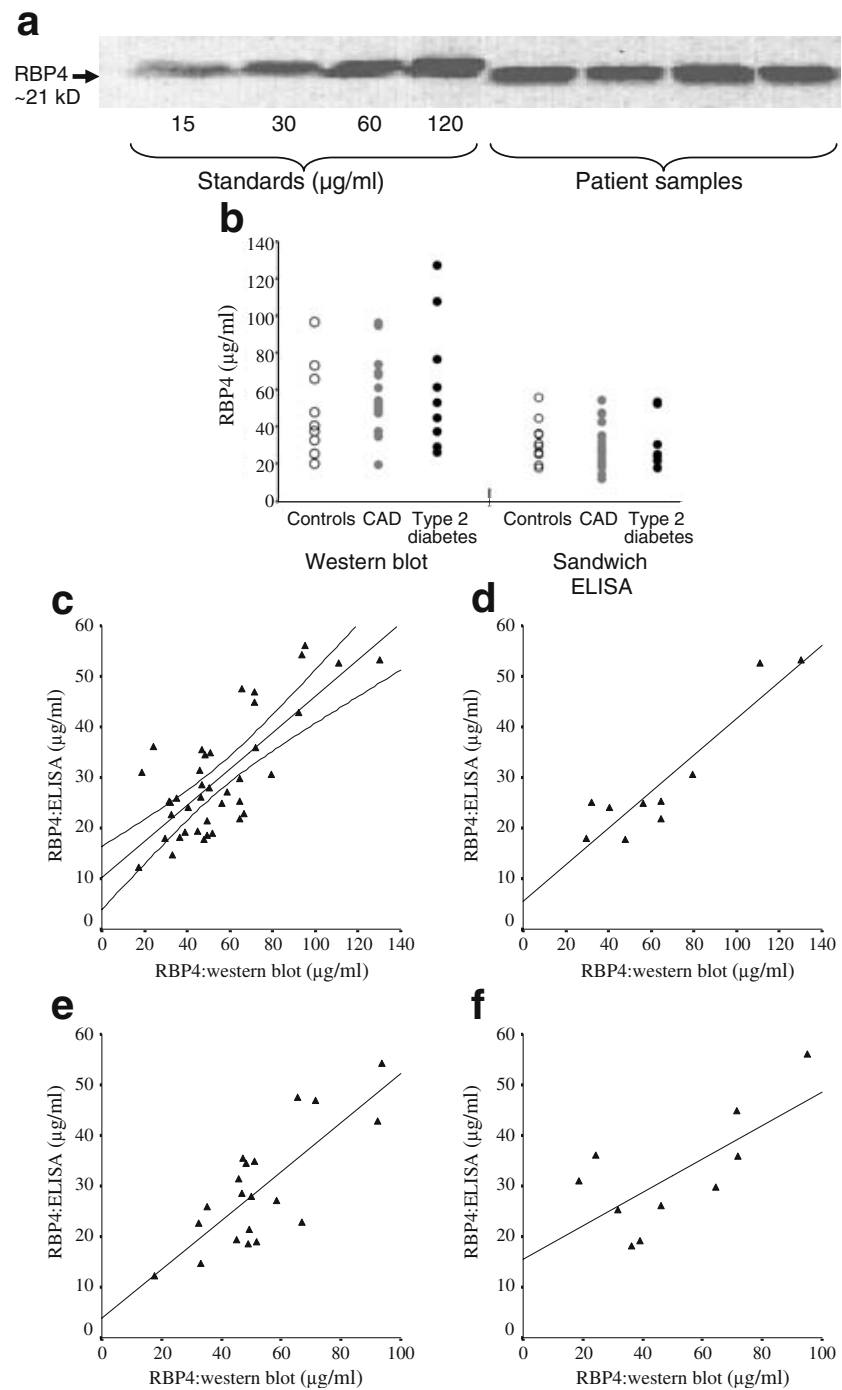

Fig. 1 a Quantitative Western blotting of serum RBP4. Serum RBP4 levels were standardised to human full-length recombinant RBP4. A single band for RBP4 migrated at $21 \mathrm{kDa}$. b Absolute measurements of RBP4 by quantitative Western blotting and commercially available sandwich ELISA as indicated. RBP4 was measured in identical serum samples. RBP4 levels were significantly higher in Western blot analysis than in ELISA [median (interquartile range)]: control subjects $46.3(31.5-71.6)$ vs $29.8(25.1-39.0) \mu \mathrm{g} / \mathrm{ml} ; p<0.001$; patients with CAD 49.1 (45.2-63.8) vs $27.8(22.5-33.9) \mu \mathrm{g} / \mathrm{ml} ; p<0.001$; type 2 diabetes $64.4(44.3-95.2)$ vs $29.5(23.7-42.9) \mu \mathrm{g} / \mathrm{ml} ; p<0.001$ However, RBP4 levels obtained by Western blot did not differ significantly between the three groups. c Linear regression analysis for RBP4 as given by Western blot and sandwich ELISA in a subgroup of 40 participants. The correlation between the two RBP4 quantification techniques was highly significant $(r=0.75 ; p<0.001)$. Significant correlations of Western blot and ELISA measurements were also found in patients with type 2 diabetes $(n=10 ; r=0.92$; $p<0.001)(\mathbf{d})$, patients with CAD $(n=20 ; r=0.80 ; p<0.001)(\mathbf{e})$ and control subjects $(n=10 ; r=0.70 ; p=0.024)(\mathbf{f})$

glycerol levels $r=0.440 ; p<0.001$; data not shown). Accordingly, we found significant correlations between serum RBP4 levels detected by quantitative Western blot and markers of lipid metabolism (total cholesterol: $r=$ 

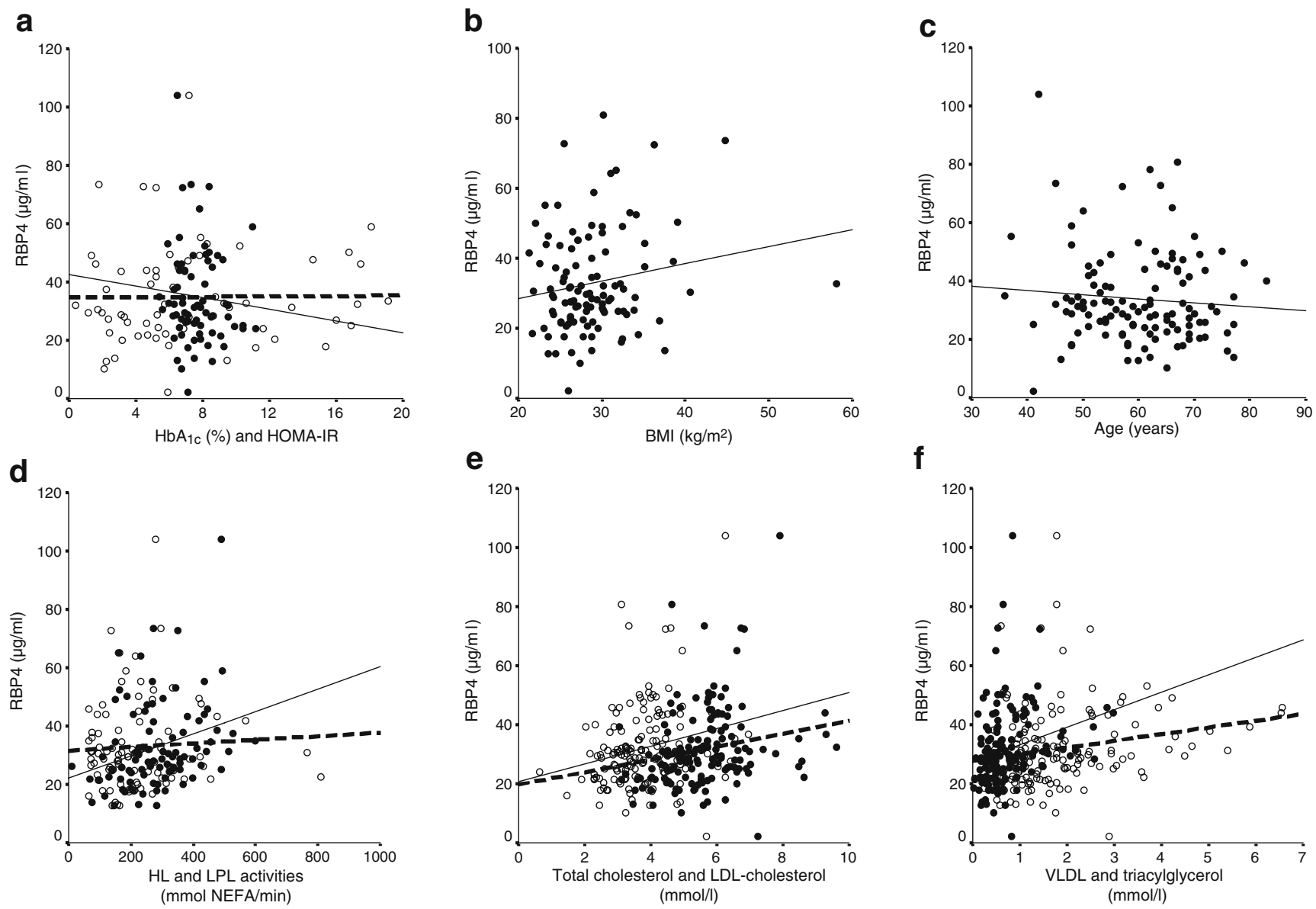

Fig. 2 Bivariate correlations of RBP4 with the following in patients with type 2 diabetes: a $\mathrm{HbA}_{1 \mathrm{c}}$ (closed circles) and HOMA-IR (open circles); b BMI; c age; d hepatic lipase (HL, closed circles) and lipoprotein lipase (LPL, open circles) activities; e total cholesterol (closed circles) and LDL-cholesterol (open circles); and f VLDLcholesterol (closed circles) and triacylglycerol (open circles). We observed significant positive correlations of HL activity $(r=0.21)$, total cholesterol $(r=0.34)$, LDL-cholesterol $(r=0.25)$, VLDL-cholesterol $(r=$ $0.35)$ and triacylglycerol $(r=0.23)$ with serum RBP4. All correlations are given as calculated by Spearman correlation coefficient. Dotted lines indicate the regression of data represented by open circles and continuous lines those of closed circles
$0.354 ; p=0.029$; VLDL-cholesterol: $r=0.429, p=0.007$; triacylglycerol levels: $r=0.533, p<0.001$ ).

To further investigate the association between serum RBP4 and circulating lipoproteins, an additional multivariable regression analysis was performed including plasma lipoproteins and the post-heparin plasma activities of hepatic lipase and lipoprotein lipase, two pivotal enzymes in lipoprotein metabolism. In type 2 diabetes patients, LDL- and VLDL-cholesterol levels were significantly and independently associated with circulating RBP4 levels. Moreover, hepatic lipase activity was independently associated with serum RBP4 (Table 3). In patients with CAD, VLDL-cholesterol and hepatic lipase activity were also independently associated with serum RBP4 levels, whereas VLDL-cholesterol, but not hepatic lipase activity showed significant independent associations with RBP4 in control subjects (Table 3 ).

\section{Discussion}

Associations of RBP4 with markers of insulin sensitivity and the metabolic syndrome To our knowledge, this is the first study to evaluate serum RBP4 levels as a marker of the metabolic state in larger groups of patients with CAD or type 2 diabetes. We demonstrated independent associations of serum RBP4 levels with pro-atherogenic lipoproteins in type 2 diabetes, patients with CAD and in healthy men without diabetes or macrovascular disease. Unexpectedly, there was no correlation of RPB4 levels with HOMA-IR in any of the groups studied, suggesting that RBP4 is not a marker of insulin sensitivity. In addition, statistical analyses of collinearity revealed that even the significant independent association between BMI and RBP4 in patients with type 2 diabetes (Table 2 ) is probably due to collinearity of BMI and both HOMA-IR and $\mathrm{HbA}_{1 \mathrm{c}}$. BMI represents a 
Fig. 3 Bivariate correlations of RBP4 with the following in patients with CAD: a HOMAIR; b BMI; c VLDL-cholesterol (closed circles) and triacylglycerol (open circles); and d hepatic lipase (HL, closed circles) and lipoprotein lipase (LPL, open circles) activities. We observed significant positive correlations of VLDL-

cholesterol $(r=0.25)$, triacylglycerol $(r=0.29)$ and HL activity $(r=0.18)$ with serum RBP4. All correlations are given as calculated by Spearman correlation coefficient. Dotted lines indicate the regression of data represented by open circles and continuous lines those of closed circles
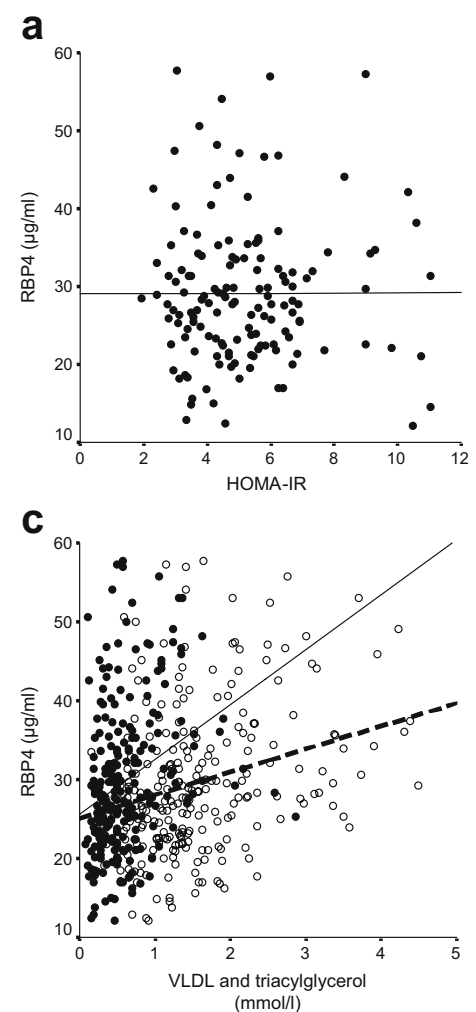
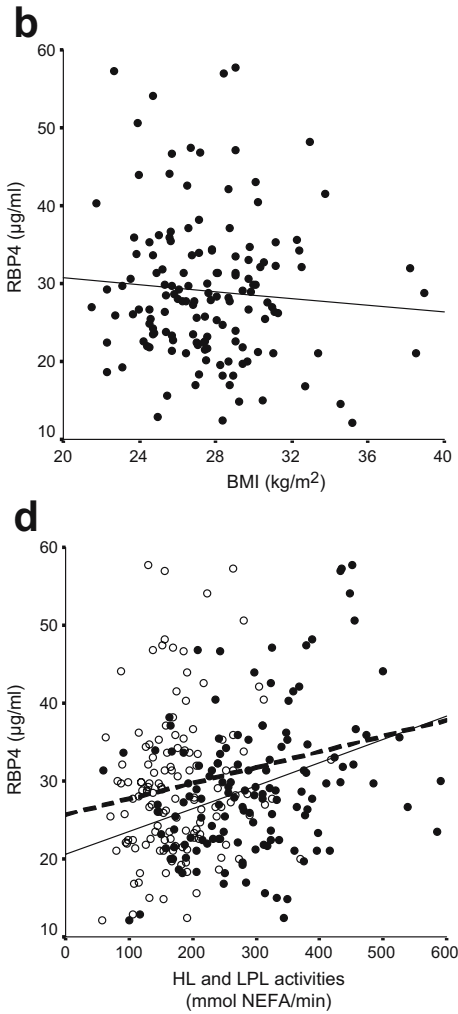

crucial anthropometric characteristic in type 2 diabetes and thus remained in the model in order to achieve applicable clinical comparability; no alterations of the significant associations were present after exclusion of BMI (data not shown).

Our findings are in accordance with recent published data [14] regarding the role of RBP4 in human obesity. In that study, RBP4 serum levels were not different between lean, overweight and obese subjects. In addition, crosssectional and weight-loss studies did not show any relationships between the HOMA-IR and adipose RBP4 expression or with circulating RBP4 concentrations [14]. This appears to be in contrast to other recently published data, which provide evidence for a connection between RBP4 and the glucose disposal rate as detected in clamp studies in men and women with type 2 diabetes, obese patients without diabetes and even in healthy subjects with a family history of diabetes [1]. The underlying reason for the divergent findings is likely to be the different design of these studies. In the present study, subjects were included according to their main clinical diagnosis and recruited during routine clinical procedures without further stratification. In the study of Graham et al. [1], the data were obtained in a more experimental setting, studying small and well defined patient groups of young age and after withdrawal of glucose-lowering medication. In contrast to the previously published data, which were only partly subjected to multivariable regression analysis, the associa- tions shown in multivariable models presented herein were adjusted for cardiovascular risk factors and the components of the metabolic syndrome. On the other hand, clamp studies reflect insulin sensitivity more accurately than the HOMA-IR used in the present study $[15,16]$. In addition, it seems possible that glucose-lowering agents in our cohort of men with type 2 diabetes might have obscured the association between the HOMA-IR and RBP4 levels.

Another factor that need to be taken into account is the differing performances of commonly used RBP4 assays, possibly causing methodological variances. A comparison of commercially available multiwell immunoassays with quantitative western blotting using full-length RBP4 protein as standard suggested that the latter is superior [8]. Yet we found close correlations between the two techniques in all patient subgroups studied. Interestingly, in accordance with the previous data [8], RBP4 levels detected by immunoblot were significantly higher than in ELISA (Fig. 1b). Additionally, linearity between the two methods was less pronounced in non-insulin-resistant control subjects. This implies the need for further experimental studies, with a view to clarifying whether multimerisation or posttranslational modifications on RBP4 impair antibody detection in the sandwich ELISA, particularly since immunoblots are performed with completely denatured and reduced proteins. 
Table 2 Independent associations between markers of the metabolic syndrome and serum RBP4

\begin{tabular}{|c|c|c|c|c|c|c|c|c|c|}
\hline \multirow[t]{2}{*}{ Variable $^{\mathrm{a}}$} & \multicolumn{3}{|c|}{ Type 2 diabetes } & \multicolumn{3}{|l|}{ CAD } & \multicolumn{3}{|c|}{ Control subjects } \\
\hline & $\beta$ & $t$ & $p$ value & $\beta$ & $t$ & $p$ value & $\beta$ & $t$ & $p$ value \\
\hline Age & 0.123 & 0.468 & 0.631 & 0.018 & 0.224 & 0.823 & -0.074 & -0.524 & 0.603 \\
\hline BMI & 0.348 & 2.279 & $0.027^{*}$ & -0.098 & -1.172 & 0.243 & 0.003 & 0.006 & 0.998 \\
\hline LDL-cholesterol & 0.268 & 1.927 & 0.060 & -0.061 & -0.765 & 0.446 & -0.187 & -1.412 & 0.164 \\
\hline VLDL-cholesterol & 0.336 & 2.274 & $0.027 *$ & 0.353 & 4.184 & $<0.001^{*}$ & 0.455 & 3.502 & $0.002 *$ \\
\hline History of hypertension & -0.011 & -0.076 & 0.939 & 0.018 & 0.234 & 0.815 & 0.220 & 1.660 & 0.104 \\
\hline HOMA-IR & -0.068 & -0.492 & 0.625 & -0.087 & -1.029 & 0.305 & 0.074 & 0.529 & 0.599 \\
\hline $\mathrm{HbA}_{1 \mathrm{c}}$ & -0.094 & -0.649 & 0.519 & - & - & - & - & - & - \\
\hline GLT & 0.157 & 1.043 & 0.302 & - & - & - & - & - & - \\
\hline$R$ & & & 0.543 & & & 0.530 & & & 0.509 \\
\hline$r^{2}$ & & & 0.295 & & & 0.281 & & & 0.259 \\
\hline
\end{tabular}

GLT, Glucose-lowering therapy

${ }^{*} p<0.05$

${ }^{a}$ RBP4, VLDL-cholesterol, HOMA-IR and $\mathrm{HbA1}_{\mathrm{c}}$ were log-transformed variables

A limitation of our present study is that we did not exclude prevalent type 2 diabetes by oral glucose tolerance testing of patients with CAD and of control subjects. Therefore, an unknown number of undiagnosed type 2 diabetes patients may have been missed, causing overlap between the three study groups. It will therefore be necessary to validate the lack of associations of RBP4 with glucose metabolism and insulin sensitivity in more specifically defined cohorts utilising sensitive measures of insulin sensitivity and glucose tolerance.

Associations between RBP4 and lipid metabolism Interestingly, we found an independent association between RBP4 and circulating lipoproteins. RBP4 levels were independently associated with pro-atherogenic VLDL- and LDLcholesterol concentrations in patients with type 2 diabetes and with VLDL-cholesterol in patients with CAD and in control subjects after adjustment for markers of the metabolic syndrome and lipid metabolism. Triacylglycerol levels, however, were not independently associated with RBP4 in the same models, suggesting a possible role for RBP4 in VLDL and LDL clearance, rather than in total plasma triacylglycerol concentration. Circulating VLDLs are hydrolysed by two lipolytic enzymes, lipoprotein lipase and hepatic lipase. Both a decrease in lipoprotein lipase and an increase in hepatic lipase activity have previously been shown to be associated with the accumulation of pro-atherogenic small dense LDL particles, a lipoprotein composition that is particularly evident in the presence of increased VLDL concentrations [17, 18]. Consistent with the above, we found an independent association between RPB4 levels and hepatic lipase activity in patients with type 2 diabetes and in those with CAD. RBP4 is a transporter protein for retinol that serves as a precursor for the synthesis of ligands of the retinoid $\mathrm{X}$ receptor and retinoic acid receptor nuclear hormone receptors [19]. In

Table 3 Independent adjusted associations between markers of lipoprotein metabolism and serum RBP4

\begin{tabular}{|c|c|c|c|c|c|c|c|c|c|}
\hline \multirow[t]{2}{*}{ Variable $^{\mathrm{a}}$} & \multicolumn{3}{|c|}{ Type 2 diabetes } & \multicolumn{3}{|l|}{ CAD } & \multicolumn{3}{|c|}{ Control subjects } \\
\hline & $\beta$ & $t$ & $p$ value & $\beta$ & $t$ & $p$ value & $\beta$ & $t$ & $p$ value \\
\hline LDL-cholesterol & 0.215 & 2.109 & $0.038^{*}$ & -0.056 & -0.781 & 0.436 & 0.118 & 0.916 & 0.364 \\
\hline VLDL-cholesterol & 0.253 & 2.416 & $0.018^{*}$ & 0.393 & 5.254 & $<0.001^{*}$ & 0.599 & 4.401 & $<0.001^{*}$ \\
\hline LPL activity & 0.116 & 1.095 & 0.277 & 0.133 & 1.928 & 0.055 & -0.039 & -0.263 & 0.794 \\
\hline HL activity & 0.247 & 2.270 & $0.026^{*}$ & 0.142 & 2.098 & $0.037^{*}$ & 0.051 & 0.415 & 0.680 \\
\hline$R$ & & & 0.448 & & & 0.400 & & & 0.562 \\
\hline$r^{2}$ & & & 0.201 & & & 0.160 & & & 0.316 \\
\hline
\end{tabular}

Associations were adjusted for age and BMI

HL, Hepatic lipase; LPL, lipoprotein lipase

${ }^{*} p<0.05$

${ }^{a}$ RBP4 and VLDL-cholesterol were log-transformed variables 
previous studies, retinoids increased the hepatic production of VLDL [20] and elevated the levels of apolipoprotein (apo) C-III [21], a hepatic protein that delays (1) the catabolism of VLDL particles by inhibiting their binding to the endothelial surface and (2) lipolysis by lipoprotein lipase [22]. This may explain the lack of an association between RBP4 and lipoprotein lipase in our study. Preliminary data derived from a subset of 41 type 2 diabetes patients in this study show a positive correlation between apoC-III and serum RBP4 ( $r=0.336 ; p<0.05$, not shown), thus strengthening the hypothesis of decreased VLDL clearance via upregulated apoC-III. Hence, RBP4 may alter the steady-state plasma VLDL- and LDL-cholesterol levels by influencing hepatic production or peripheral clearance or both.

In conclusion, RBP4 does not seem to be a valuable marker for identification of the metabolic syndrome and insulin resistance in male patients with diabetes or CAD under clinical settings. Independent associations of RBP4 with pro-atherogenic lipoproteins and key enzymes of lipoprotein metabolism in patients with and without the metabolic syndrome indicate that RBP4 may play a role in lipid metabolism. This needs to be further investigated in experimental follow-up studies.

Acknowledgement The study was partly supported by the Lautenschläger Stiftung (Foundation) (to P. P. Nawroth).

Duality of interest The authors declare that there is no duality of interest associated with this manuscript.

\section{References}

1. Graham TE, Yang Q, Bluher M et al (2006) Retinol-binding protein 4 and insulin resistance in lean, obese, and diabetic subjects. N Engl J Med 354:2552-2563

2. Yang Q, Graham TE, Mody N et al (2005) Serum retinol binding protein 4 contributes to insulin resistance in obesity and type 2 diabetes. Nature 436:356-362

3. Garvey WT, Maianu L, Huecksteadt TP, Birnbaum MJ, Molina JM, Ciaraldi TP (1991) Pretranslational suppression of a glucose transporter protein causes insulin resistance in adipocytes from patients with non-insulin-dependent diabetes mellitus and obesity. J Clin Invest 87:1072-1081

4. Shepherd PR, Kahn BB (1999) Glucose transporters and insulin action-implications for insulin resistance and diabetes mellitus. N Engl J Med 341:248-257

5. DeFronzo RA, Ferrannini E (1991) Insulin resistance. A multifaceted syndrome responsible for NIDDM, obesity, hypertension, dyslipidemia, and atherosclerotic cardiovascular disease. Diabetes Care 14:173-194

6. Isomaa B, Almgren P, Tuomi T et al (2001) Cardiovascular morbidity and mortality associated with the metabolic syndrome. Diabetes Care 24:683-689

7. Expert Committee on the Diagnosis and Classification of Diabetes Mellitus (1997) Report of the Expert Committee on the Diagnosis and Classification of Diabetes Mellitus. Diabetes Care 20:1183-1197

8. Graham TE, Wason M, Bluher M, Kahn BB (2007) Shortcomings in methodology complicate measurements of serum retinol binding protein (RBP4) in insulin-resistant human subjects. Diabetologia 50:814-823

9. Levy JC, Matthews DR, Hermans MP (1998) Correct homeostasis model assessment (HOMA) evaluation uses the computer program. Diabetes Care 21:2191-2192

10. Iverius PH, Brunzell JD (1985) Human adipose tissue lipoprotein lipase: changes with feeding and relation to postheparin plasma enzyme. Am J Physiol 249:E107-E114

11. Schneider JG, von Eynatten M, Schiekofer S, Nawroth PP, Dugi KA (2005) Low plasma adiponectin levels are associated with increased hepatic lipase activity in vivo. Diabetes Care 28:2181-2186

12. von Eynatten M, Schneider JG, Humpert PM et al (2004) Decreased plasma lipoprotein lipase in hypoadiponectinemia: an association independent of systemic inflammation and insulin resistance. Diabetes Care 27:2925-2929

13. Trujillo ME, Scherer PE (2005) Adiponectin-journey from an adipocyte secretory protein to biomarker of the metabolic syndrome. J Intern Med 257:167-175

14. Janke J, Engeli S, Boschmann M et al (2006) Retinol-binding protein 4 in human obesity. Diabetes 55:2805-2810

15. Kang ES, Yun YS, Park SW et al (2005) Limitation of the validity of the homeostasis model assessment as an index of insulin resistance in Korea. Metabolism 54:206-211

16. Uwaifo GI, Fallon EM, Chin J, Elberg J, Parikh SJ, Yanovski JA (2002) Indices of insulin action, disposal, and secretion derived from fasting samples and clamps in normal glucose-tolerant black and white children. Diabetes Care 25:2081-2087

17. Zambon A, Austin MA, Brown BG, Hokanson JE, Brunzell JD (1993) Effect of hepatic lipase on LDL in normal men and those with coronary artery disease. Arterioscler Thromb 13:147-153

18. Patsch JR, Prasad S, Gotto AM Jr, Patsch W (1987) High density lipoprotein2. Relationship of the plasma levels of this lipoprotein species to its composition, to the magnitude of postprandial lipemia, and to the activities of lipoprotein lipase and hepatic lipase. J Clin Invest 80:341-347

19. Marill J, Idres N, Capron CC, Nguyen E, Chabot GG (2003) Retinoic acid metabolism and mechanism of action: a review. Curr Drug Metab 4:1-10

20. Gerber LE, Erdman JW Jr (1981) Retinoic acid and hypertriglyceridemia. Ann N Y Acad Sci 359:391-392

21. Vu-Dac N, Gervois P, Torra IP et al (1998) Retinoids increase human apo C-III expression at the transcriptional level via the retinoid $\mathrm{X}$ receptor. Contribution to the hypertriglyceridemic action of retinoids. J Clin Invest 102:625-632

22. Aalto-Setala K, Fisher EA, Chen X et al (1992) Mechanism of hypertriglyceridemia in human apolipoprotein (apo) CIII transgenic mice. Diminished very low density lipoprotein fractional catabolic rate associated with increased apo CIII and reduced apo E on the particles. J Clin Invest 90:18891900 